\title{
Novel Application of Magnetite Nanoparticle-Mediated Vitamin D3 Delivery for Peritoneal Dialysis-Related Peritoneal Damage
}

This article was published in the following Dove Press journal: International Journal of Nanomedicine

\author{
Fong-Yu Cheng (D) \\ Yuan-Yow Chiou (iD ${ }^{2,3}$ \\ Shih-Yuan Hung ${ }^{4,5}$ \\ Tsun-Mei Lin ${ }^{6}$ \\ Hao-Kuang Wang $\mathbb{D}^{7}$ \\ Chi-Wei Lin $^{8}$ \\ Hung-Hsiang Liou ${ }^{9}$ \\ Min-Yu Chang (iD ${ }^{5}$ \\ Hsi-Hao Wang ${ }^{5}$ \\ Yi-Che Lee (iD) ${ }^{4,5,10}$
}

'Department of Chemistry, Chinese Culture University, Taipei, Taiwan;

${ }^{2}$ Department of Pediatrics, National Cheng Kung University Hospital, Tainan,

Taiwan; ${ }^{3}$ Institute of Clinical Medicine, College of Medicine, National Cheng

Kung University, Tainan, Taiwan; ${ }^{4}$ School of Medicine, College of Medicine, I-Shou University, Kaohsiung, Taiwan; ${ }^{5}$ Division of Nephrology, Department of Internal Medicine, E-DA Hospital, Kaohsiung, Taiwan; 'Department of Laboratory Medicine, E-DA Hospital, Kaohsiung, Taiwan; ${ }^{7}$ Department of Neurosurgery, E-DA Hospital, Kaohsiung, Taiwan;

${ }^{8}$ Department of Medical Education, E-DA Hospital, Kaohsiung, Taiwan; ${ }^{9}$ Division of Nephrology, Department of Internal Medicine, Hsin-Jen Hospital, New Taipei City, Taiwan; ${ }^{10}$ Division of Nephrology, Department of Internal Medicine, E-DA Dachang Hospital, Kaohsiung, Taiwan

Correspondence: Yi-Che Lee Division of Nephrology, Department of Internal Medicine, E-DA Hospital//-Shou University, No. I, Yida Road, Jiaosu Village, Yanchao District, Kaohsiung City, 82445,

Taiwan, R.O.C

Tel +886-7-6I500I I Ext 5I2I

Email pipijer@gmail.com
Purpose: Vitamin D3 is useful for the treatment of peritoneal dialysis (PD)-related peritoneal damage, but its side effects, such as hypercalcemia and vascular calcification, limit its applicability. Thus, we developed vitamin D-loaded magnetic nanoparticles (MNPs) and determined their therapeutic efficacy and side effects in vivo.

Materials and Methods: Alginate-modified MNPs were combined with $1 \alpha, 25(\mathrm{OH})_{2} \mathrm{D}_{3}$ to generate vitamin D-loaded nanoparticles. The particles were conjugated with an antibody against peritoneum-glycoprotein M6A (GPM6A). The particles' ability to target the peritoneum was examined following intraperitoneal administration to mice and by monitoring their bio-distribution. We also established a PD animal model to determine the therapeutic and side effects of vitamin D-loaded MNPs in vivo.

Results: Vitamin D-loaded MNPs targeted the peritoneum better than vitamin D3, and had the same therapeutic effect as vitamin D3 in ameliorating peritoneal fibrosis and functional deterioration in a PD animal model. Most importantly, the particles reduced the side effects of vitamin D3, such as hypercalcemia and body weight loss, in mice.

Conclusion: Vitamin D-loaded MNPs could be an ideal future therapeutic option to treat PD-related peritoneal damage.

Keywords: peritoneal dialysis, nanoparticles, vitamin D, fibrosis

\section{Introduction}

Peritoneal dialysis (PD) is an important renal replacement therapy for end-stage renal disease (ESRD). However, peritoneal injury and technique failure are common PD complications. ${ }^{1-6}$ Peritoneal injury is mostly attributed to bio-incompatible dialysate and frequently occurs during PD therapy. ${ }^{2,5,7-10}$

Nanotechnology research has shown that nanoparticles (NPs) can serve as good drug carriers. Targeted nano-drug delivery systems (nano-DDS) can deliver drugs specifically to the target site, ensuring site-specific activity. Nano-DDS can also prevent drug degradation, thus ensuring a higher drug concentration at the target site, which in turn may reduce drug dosage. ${ }^{11}$ This is particularly important for drugs with a marginal difference between their therapeutic and toxic concentrations, so that the side effects can be minimized.

It has been shown that vitamin D3 can be used to treat peritoneal damage induced by PD therapy. ${ }^{3,12}$ However, its clinical application is limited due to side effects such as hypercalcemia, hyperphosphatemia, and vascular calcification. To 
overcome the side effects and poor water solubility of hydrophobic drugs such as vitamin D3, nanomaterials are commonly used as drug carriers because of their enhanced accumulation ability at the target region. In our previous study, we constructed vitamin D-liposomal NPs and examined their therapeutic effects in vitro. ${ }^{13}$ The results showed that these NPs were taken up by mesothelial cells and did not cause cell toxicity as well as provided the same therapeutic effect as vitamin D3. However, the therapeutic effects of these NPs in vivo are still unknown. Therefore, in this follow-up study, a magnetite $\left(\mathrm{Fe}_{3} \mathrm{O}_{4}\right)$ magnetic nanoparticle (MNP) was selected as the drug carrier to fabricate vitamin D-loaded MNPs. The main reasons for selecting $\mathrm{Fe}_{3} \mathrm{O}_{4} \mathrm{NP}$ are its safety (an FDA-approved material for human use) and its ability to conjugate with alginate to encapsulate vitamin D3. Then we investigated the therapeutic effect of vitamin D-loaded MNPs in PD animal model.

\section{Materials and Methods}

\section{Preparation of Vitamin D-Loaded}

\section{Magnetic NPs (Vit.D-MNPs)}

The alginate-modified magnetic NPs (alg-MNPs) were prepared as described previously. ${ }^{14}$ Vitamin D3 was dissolved in DMSO at a concentration of $5 \mathrm{mg} / \mathrm{mL}$. AlgMNPs $(100 \mu \mathrm{L}$; Fe concentration: $2.4 \mu \mathrm{g} / \mu \mathrm{L})$ were added to $900 \mu \mathrm{L}$ acetone and centrifuged at $15,000 \mathrm{rpm}$ for 5 min. After the supernatant was removed, $100 \mu \mathrm{L}$ vitamin D3 solution was added and then sonicated until all the precipitate dissolved in DMSO. Subsequently, $200 \mu \mathrm{L}$ of distilled water was added to the resulting solution and incubated for $5 \mathrm{~min}$ at room temperature. Finally, $40 \mu \mathrm{L}$ calcium chloride $(0.1 \mathrm{M})$ was added, and the solution was mixed and incubated for 2 min (Supplementary Figure 1). The supernatant was collected by magnetic separation, and the precipitate was redispersed in $800 \mu \mathrm{L}$ distilled water by sonication. Vitamin D3 concentration in the supernatant was measured by high-performance liquid chromatography (HPLC) to calculate the loading efficiency of vitamin D3. The precipitate aqueous dispersion was stored at $4{ }^{\circ} \mathrm{C}$ in the dark for further use.

\section{Synthesis of Rhodamine 6G-Loaded MNPs (R6G-MNPs)}

R6G was dissolved in DMSO at a concentration of $1 \mathrm{mM}$. Alg-MNPs (100 $\mu \mathrm{L}$; Fe concentration: $2.4 \mu \mathrm{g} / \mu \mathrm{L})$ were added to $900 \mu \mathrm{L}$ acetone and centrifuged at 15,000 rpm for
$5 \mathrm{~min}$. After the supernatant was removed, $100 \mu \mathrm{L}$ R6G solution was added and sonicated until all the precipitate (R6G-loaded MNPs) dissolved. The rest of the process was the same as described above. R6G-loaded MNPs were stored at $4{ }^{\circ} \mathrm{C}$ in the dark until further use.

\section{Nanoparticle (NP) Conjugation with Glycoprotein M6A Antibody (Ab-Vit. D-MNPs)}

To enhance the uptake of Vit.D-MNPs by the peritoneum, the NPs were conjugated with a peritoneum-glycoprotein M6A (GPM6A) antibody. GPM6A antibody (MBL) (10 nmol) was mixed with $1 \mathrm{nmol}$ NPs in $0.5 \mathrm{~mL}$ distilled water; next, $1.5 \mathrm{nmol} \mathrm{N}$-Ethyl-N'-(3-dimethylaminopropyl) carbodiimide hydrochloride was added and the mixture was incubated for $30 \mathrm{~min}$. The supernatant was removed, and the precipitate (GPM6A-conjugated MNPs) was collected by magnetic separation. The precipitate was redispersed in distilled water and stored at $4{ }^{\circ} \mathrm{C}$ for further use.

\section{General Procedures for the Quantification of Vitamin D3 Loading} Vitamin-D3 loading was determined using HPLC (Agilent 1260 Infinity system), along with a ZORBAX Eclipse PAH polymeric $\mathrm{C} 18$ bonded column (Agilent); methanol (J. T. Baker) and water $(92: 8 \% \mathrm{v} / \mathrm{v})$ as the mobile phase. The other conditions were as follows: flow rate of $2 \mathrm{~mL} /$ min, column temperature of $40{ }^{\circ} \mathrm{C}$, and a variable wavelength detector (VWD) recognition at $280 \mathrm{~nm}^{15}$ A calibration curve for $1 \alpha, 25(\mathrm{OH})_{2} \mathrm{D}_{3}$ was constructed in the concentration range of $0.05-0.5 \mathrm{mg} / \mathrm{mL}$ by diluting $1 \mathrm{mg} / \mathrm{mL}$ of the standard stock solution in methanol.

The loading efficiency of vitamin D3 was calculated from the difference between the initial amount of vitamin D3 $\left(D_{i}\right)$ and the total amount of free vitamin D3 in the supernatant $\left(\mathrm{D}_{\mathrm{s}}\right)$.

Loading efficiency $(\%)=\left(D_{\mathrm{i}}-\mathrm{D}_{\mathrm{s}}\right) / \mathrm{D}_{\mathrm{s}} \times 100 \%$

\section{General Procedure for Determining Vitamin D3 Release Profile}

Vit.D-MNPs $(200 \mu \mathrm{L})$ were mixed with $300 \mu \mathrm{L}$ PBS buffer and incubated for $2 \mathrm{~h}$ at room temperature. After brief centrifugation at $10,000 \mathrm{rpm}$, the supernatant was collected by magnetic separation and retained for subsequent HPLC analysis. PBS buffer ( $300 \mu \mathrm{L})$ was added to the residue in the original tube and sonicated. The mixture 
was briefly centrifuged, and the supernatant was collected and used as previously described. After collecting all the supernatants at $2,4,8,24,48$, and $72 \mathrm{~h}$, the volume was recorded and the concentration of vitamin D3 was determined by HPLC. The final release profile of vitamin D3 was determined by calculating the cumulative amount of vitamin D3 released over time.

\section{Transmission Electron Microscopy (TEM) of Nanoparticles (NPs)}

Suspensions of NPs in $\mathrm{H}_{2} \mathrm{O}$ were mixed with uranyl acetate for staining studies. Ten microliters of this solution was then transferred to a TEM grid (copper grid, $3.0 \mathrm{~mm}, 200$ mesh, coated with Formvar film) and allowed to dry. The stained grids were analyzed using a JEOL JEM 2100 (Tokyo, Japan) TEM.

\section{Observing Nanoparticles (NPs)}

\section{Conjugated with Glycoprotein M6A Antibody Using an in vivo Imaging System (IVIS)}

All mouse experiments were approved by the Laboratory Animal Center of the National Cheng Kung University and were handled according to the "Guide for The Care and
Use of Laboratory Animals" (NRC, USA 2011). Four- to six-week-old syngeneic C57BL/6 male mice were randomly separated into three groups. A standard diet and free access to water were provided. To assay NP biodistribution in vivo, R6G-MNPs alone or conjugated with the GPM6A antibody (R6G-MNPs/Ab-R6G-MNPs) were injected intraperitoneally (i.p.) once at a volume of 500 $\mu \mathrm{L} /$ mouse. R6G fluorescence was monitored after 6 $\mathrm{h}$ using IVIS at a wavelength of 535-580 nm. Mice in the control group were injected with $500 \mu \mathrm{L}$ autoclaved PBS per mouse. The animals were sacrificed after 72 h. Tissues were collected, kept in the dark, and examined by IVIS to quantify fluorescence. Finally, all tissues were dissolved by adding nitric acid $\left(\mathrm{HNO}_{3}\right)$ and hydrochloric acid $(\mathrm{HCl})$. The amount of $\mathrm{Fe}$ in the parietal peritoneum, mesentery, liver, kidney, and urine were also measured by an atomic absorption (AA) instrument.

\section{Peritoneal Fibrosis Model: Experimental Protocol}

To investigate the protective effect of Ab-Vit.D-MNPs in a peritoneal dialysis model, 7-8-week-old C57BL/6 male mice were used. Peritoneal fibrosis was induced by using chlorhexidine gluconate $(\mathrm{CG})$ based on a modified procedure
MNPs

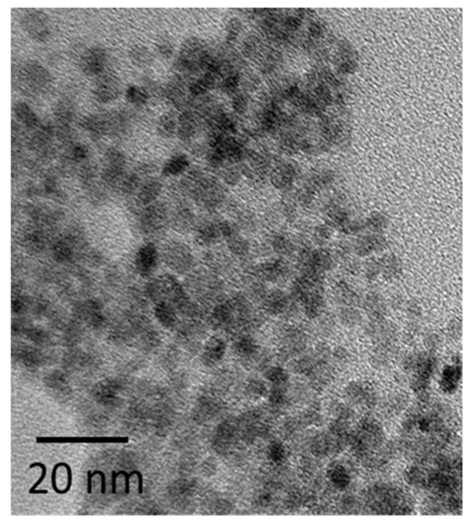

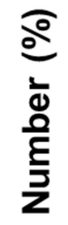

Diameter $98.2 \mathrm{~nm}$
Vit.D-MNPs
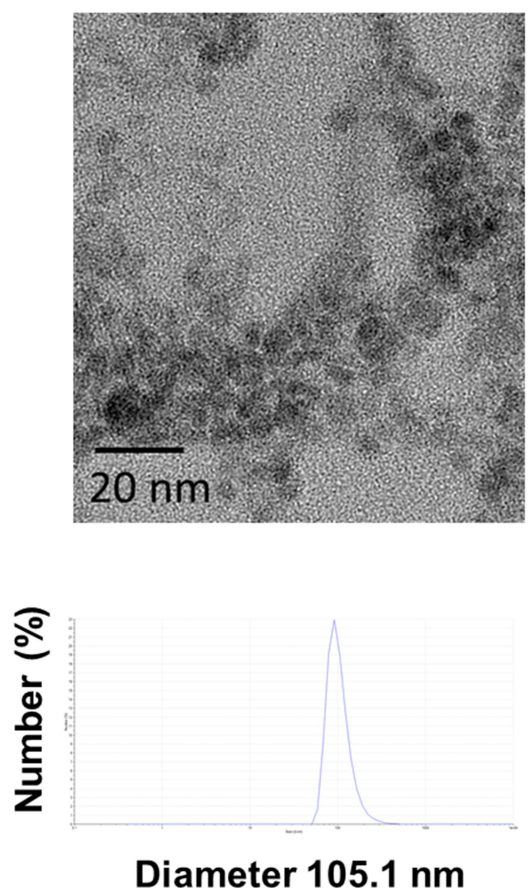

Ab-Vit.D-MNPs
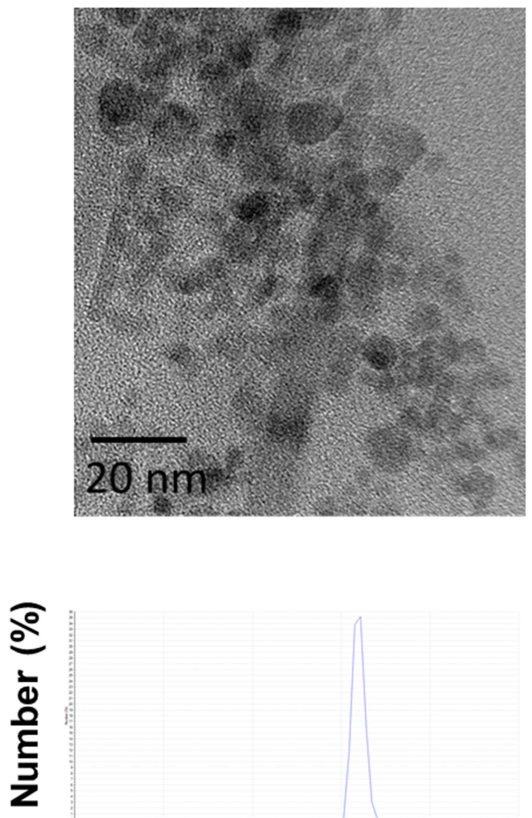

Diameter 168.4 nm

Figure I Characterization and size distribution of alginate-modified magnetic nanoparticles (alg-MNPs), vitamin D-loaded MNPs (Vit.D-MNPs), and Ab-Vit.D-MNPs by transmission electron microscopy (TEM) imaging and differential light scattering (DLS). 


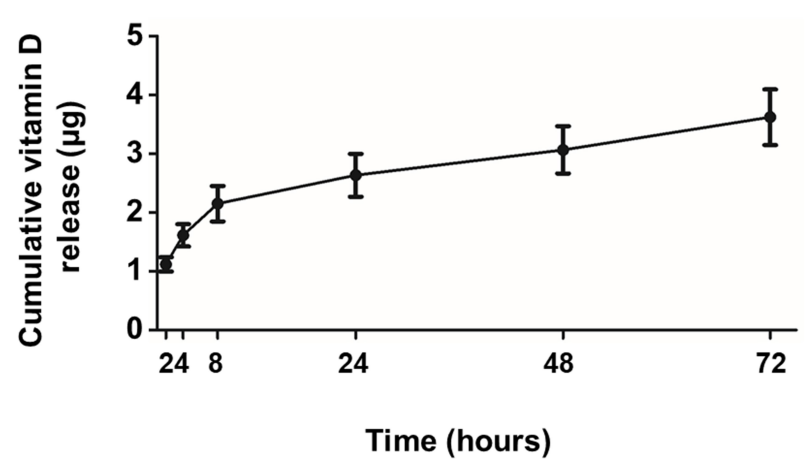

Figure 2 Release profile of vitamin $D$ from vitamin D-loaded magnetic nanoparticles (Vit.D-MNPs) over 72 hours. From the release profile, the Vit.D-MNPs appear to release vitamin $D$ in a slow and sustained manner.

of Ishii et al. ${ }^{16}$ Briefly, mice received i.p. injections of $0.1 \%$ $\mathrm{CG}$ in $15 \%$ ethanol in normal saline or normal saline alone (control) at a dose of $12.5 \mu \mathrm{L} / \mathrm{g}$ body weight (BW) every other day (QOD) for 3 weeks (9 times in total). In the group receiving $C G+$ vitamin $D$, mice received QOD IP injections of CG and daily i.p. injections of vitamin D3 $(0.004 \mu \mathrm{g} / \mathrm{g} \mathrm{BW})$ for a total of 3 weeks. In the group receiving $\mathrm{CG}+\mathrm{MNPs}$ only, mice received QOD IP injections of CG and daily i.p. injection of alg-MNPs without vitamin D3 for a total of 3 weeks. In the group receiving $\mathrm{CG}$ with $\mathrm{Ab}-\mathrm{Vit} . \mathrm{D}-\mathrm{MNPs}$, mice received QOD IP injections of $\mathrm{CG}$ and daily i.p. injection of Vit. D-MNPs $(0.004 \mu \mathrm{g} / \mathrm{g}$ BW of vitamin D3) for a total of 3 weeks (each group $\mathrm{n}=6$ ). Finally, a modified peritoneal equilibration test was performed 21 days after the first i.p. injection, and a blood sample was obtained via cardiac puncture. $^{17}$

\section{Histopathological Examination}

The liver peritoneum was fixed overnight in $4 \%$ buffered formaldehyde, processed by standard methods, and stained with Masson's trichrome. Liver surfaces were evaluated using morphometry. The peritoneum thickness was measured and expressed as the mean \pm standard deviation (SD). For each mouse, the corresponding samples were measured at 10 random sites in a blinded fashion by researchers performing microscopic analysis employing ocular metrics.

\section{Modified 30-Min Peritoneal Equilibration Test}

To evaluate the peritoneal ultrafiltration rate, mice were anesthetized (Zoletil 50: Rompun $20=1$ : 2; dose $20 \mu \mathrm{L} /$ $20 \mathrm{~g} \mathrm{BW}$ for $1 \mathrm{~h}$; intramuscular injection) and instilled with $2 \mathrm{~mL} / 20 \mathrm{~g} \mathrm{BW}$ of commercial dialysis solution containing $\quad 4.25 \%$ glucose (Dianeal; Baxter International, Inc., IL, USA). After $30 \mathrm{~min}$, the peritoneal fluid was collected, and the residual volume was recorded. Net ultrafiltration was calculated using the formula: (final dialysate volume - initial dialysate volume)/initial dialysate volume. Mass transfer of glucose (MTG) was calculated using the following formula: (initial dialysate glucose $\times$ initial volume) - (final dialysate glucose $\times$ final volume). ${ }^{17}$

\section{Statistical Analysis}

All data are expressed as the mean \pm SD. Statistical significance was analyzed with a one-way analysis of variance (ANOVA) followed by post hoc Bonferroni test. A significant result was defined as $\mathrm{P}<0.05$. All statistical analyses were performed using GraphPad Prism 8.0. (GraphPad Software, Inc., CA, US).

\section{Results}

\section{Characterization of Glycoprotein M6A Antibody-Vitamin D Nanoparticles (Ab-Vit.D-MNPs)}

We constructed nanocomposites from alg-MNPs, vitamin D3, and fluorescent dye. (Supplementary Figure 2). The real size and hydrodynamic diameters of the algMNPs, Vit.D-MNPs, and Ab-Vit.D-MNPs were determined by TEM and dynamic light scattering (DLS) (Figure 1), respectively. The real and hydrodynamic diameters of the alg-MNPs were $7.2 \pm 1.1 \mathrm{~nm}$ and $\sim 98.2 \mathrm{~nm}$ (polydispersity index: 0.31), respectively. The large hydrodynamic diameter of alg-MNPs was due to the alginate present on the particle surface. To fabricate the Vit.D-MNPs, the NP surface alginate was chelated by $\mathrm{Ca}^{2+}$, and vitamin $\mathrm{D}$ was then trapped inside the NPs. The real and hydrodynamic diameter of the Vit.D-MNPs was $\sim 7.4 \pm 1.3 \mathrm{~nm}$ and $\sim 105.1 \mathrm{~nm}$ (polydispersity index: 0.36), respectively. The change in the hydrodynamic diameter from 98.2 to $105.1 \mathrm{~nm}$ was due to encapsulation of Vitamin D in alg-MNPs and the presence of $\mathrm{Ca}^{2+}$ on the particle surface. Finally, the real and hydrodynamic diameters of Ab-Vit.D-MNPs were $\sim 7.6 \pm 1.2 \mathrm{~nm}$ and $168.4 \mathrm{~nm}$ (polydispersity index: 0.40), respectively. The large hydrodynamic diameter increase from $\sim 105.1$ to $\sim 168.4 \mathrm{~nm}$ indicated that the antibody was conjugated to the particle surface. The DLS and TEM data showed that Ab-Vit.D-MNPs formed dispersed and sub-200 $\mathrm{nm}$ spherical particles, 
A
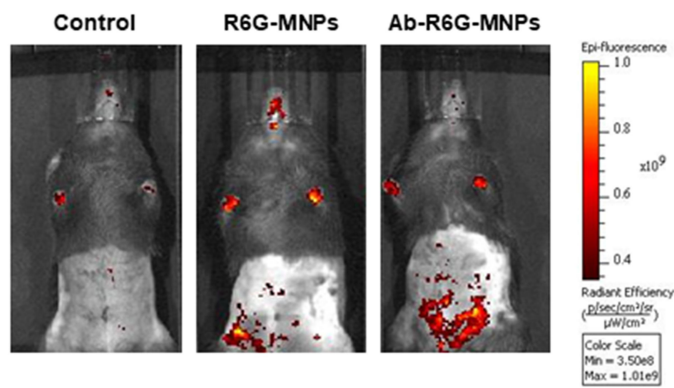

C

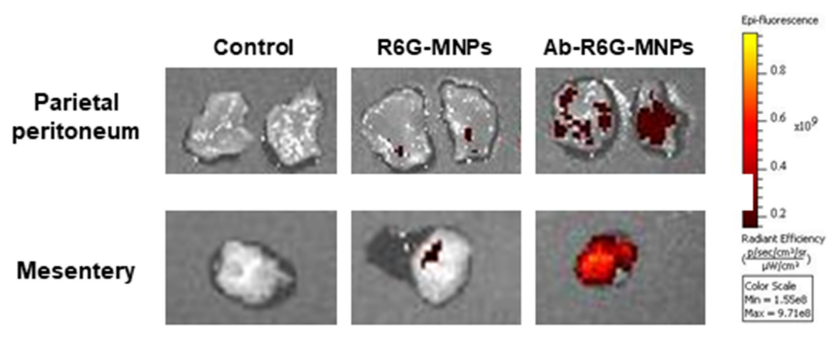

B

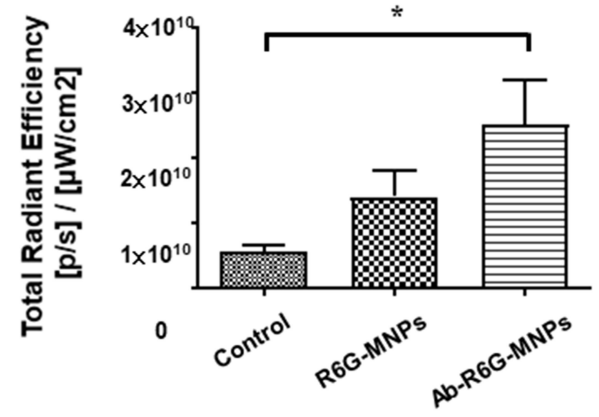

D

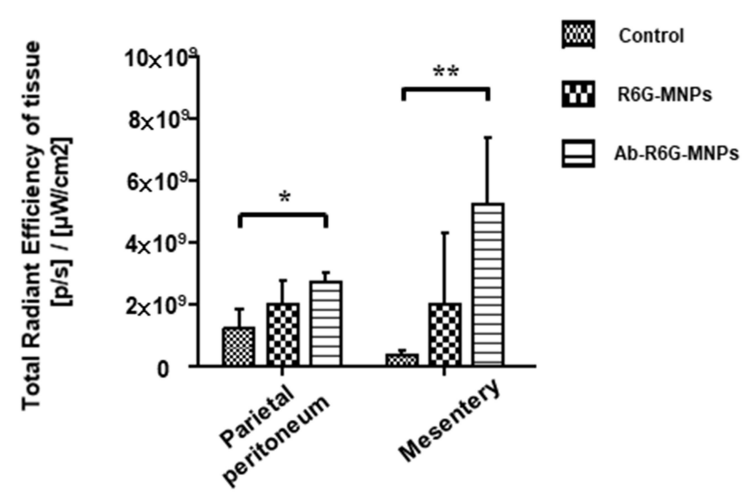

E

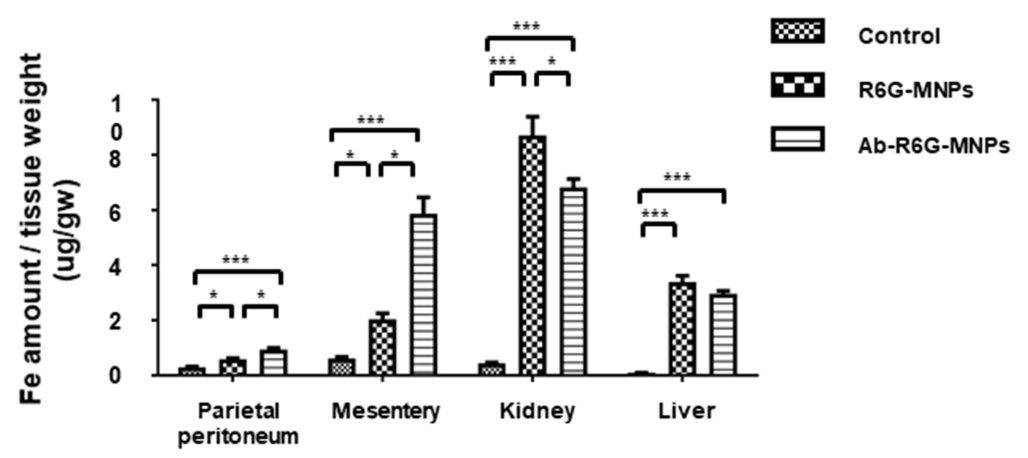

Figure 3 GPM6A antibody conjugated nanoparticles (NPs) allow enhanced peritoneal uptake in vivo. Mice were injected intraperitoneally (i.p.) with R6G-MNPs and Ab-R6G -MNPs. Using in vivo imaging system (IVIS), an enhanced Ab-R6G-MNPs presence in the peritoneum was seen 6 hours after injection, suggesting enhanced peritoneal uptake $(n \geq 3)$ (A and B). 72 hours after injection, mice were sacrificed, and tissue fluorescence measurements showed an increase in the Ab-R6G-MNP group ( $\geq 3$ ) (C and $\mathbf{D})$. The Fe in the parietal peritoneum and mesentery tissue was significantly increased in the Ab-R6G-MNP group $72 \mathrm{~h}$ after injection ( $\mathrm{n} \geq 6$ ) (E) (Data are represented as the mean $\pm \mathrm{SD}$; $* \mathrm{P}<0.05$, **P $<0.01$, *** $\mathrm{P}<0.001$ ).

ideal for enhanced permeability and retention. ${ }^{18}$ The loading efficiency of Vit.D-MNPs was calculated to be $\sim 95 \%$ based on HPLC data (Supplementary Figure 3).

\section{Release Profile of Vitamin D3 from Nanoparticles (NPs)}

To assess the release profile of vitamin D, Vit.D-MNPs were incubated in sterile PBS and isolated using a centrifugation-and-magnetic-separation method. At 2-, 4-, 8-, 24-, 48-, and 72-hour time points, each tube was shortly centrifuged, and the supernatant was collected by magnetic separation. All supernatants were analyzed by HPLC to determine the free vitamin D3 concentration (Figure 2). The results showed that $1 \alpha$, $25(\mathrm{OH})_{2} \mathrm{D}_{3}$ was released from NPs in a slow and sustained manner. 


\section{A}

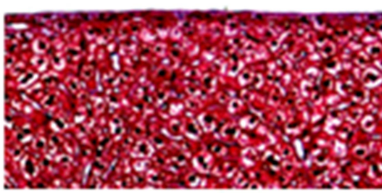

NS

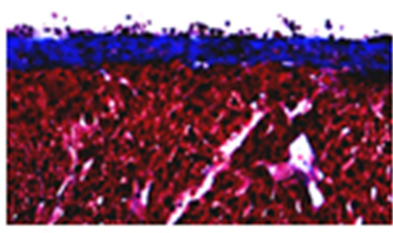

CG

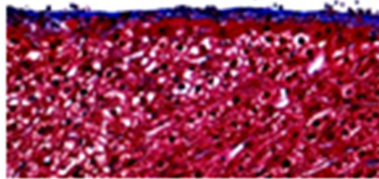

CG + Vit.D

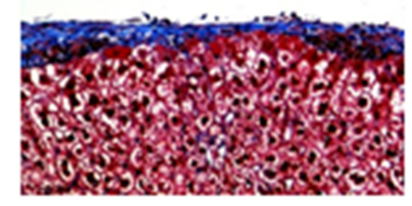

CG + MNPs only

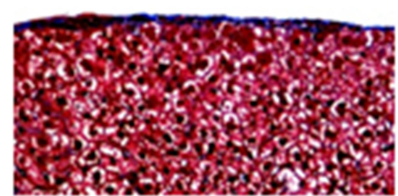

CG + Ab-Vit.D-MNPs

B

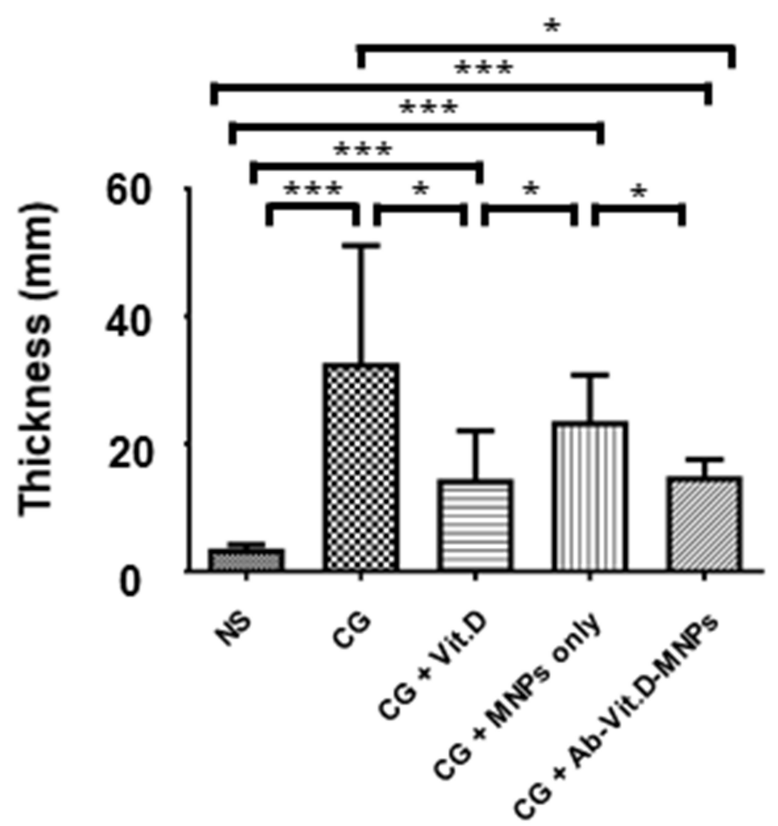

Figure $4 \mathrm{Ab}$-Vit.D-MNPs have the same therapeutic effect as vitamin D3 in ameliorating peritoneal fibrosis in a peritoneal dialysis (PD) mouse model. (A and B) Peritoneal fibrosis in the chlorhexidine gluconate (CG)-exposed group was significantly more severe than that in the saline group. Vitamin D3 significantly ameliorated peritoneal fibrosis, as visualized by Masson's trichrome staining. Ab-Vit.D-MNPs have the same therapeutic effect on peritoneal fibrosis as vitamin D (Data are represented as the mean $\pm \mathrm{SD}, \mathrm{n} \geq 3 ; * \mathrm{P}<0.05$, ***P $<0.001)$.

\section{GPM6A Antibody-Conjugated} Nanoparticles (NPs) Reach Specific Peritoneum Target in vivo

Mice were injected i.p. with $500 \mu \mathrm{L}$ R6G-MNPs and AbR6G-MNPs. The IVIS spectrum showed an enhanced peritoneal particle presence in the Ab-R6G-MNP group 6 $\mathrm{h}$ after injection, suggesting a peritoneum-targeting effect (Figure $3 \mathrm{~A}$ and $\mathrm{B})(\mathrm{n}=3$ in each group). Mice were sacrificed $72 \mathrm{~h}$ after injection. Tissue fluorescence at that time point showed that this enhancement was still sustained (Figure $3 \mathrm{C}$ and $\mathrm{D}$ ). Figure $3 \mathrm{E}$ showed that the $\mathrm{Fe}$ in the parietal peritoneum and mesentery tissue was significantly increased in the Ab-R6G-MNP group $72 \mathrm{~h}$ after injection ( $\mathrm{n} \geq 6$ in each group). The spot urine (bladder puncture) Fe content $72 \mathrm{~h}$ after injection showed nanoparticles are mainly excreted through the kidneys (Supplementary Figure 4). Besides, there was no renal or liver toxicity (Supplementary Figures 5 and $\underline{6}$ ). 

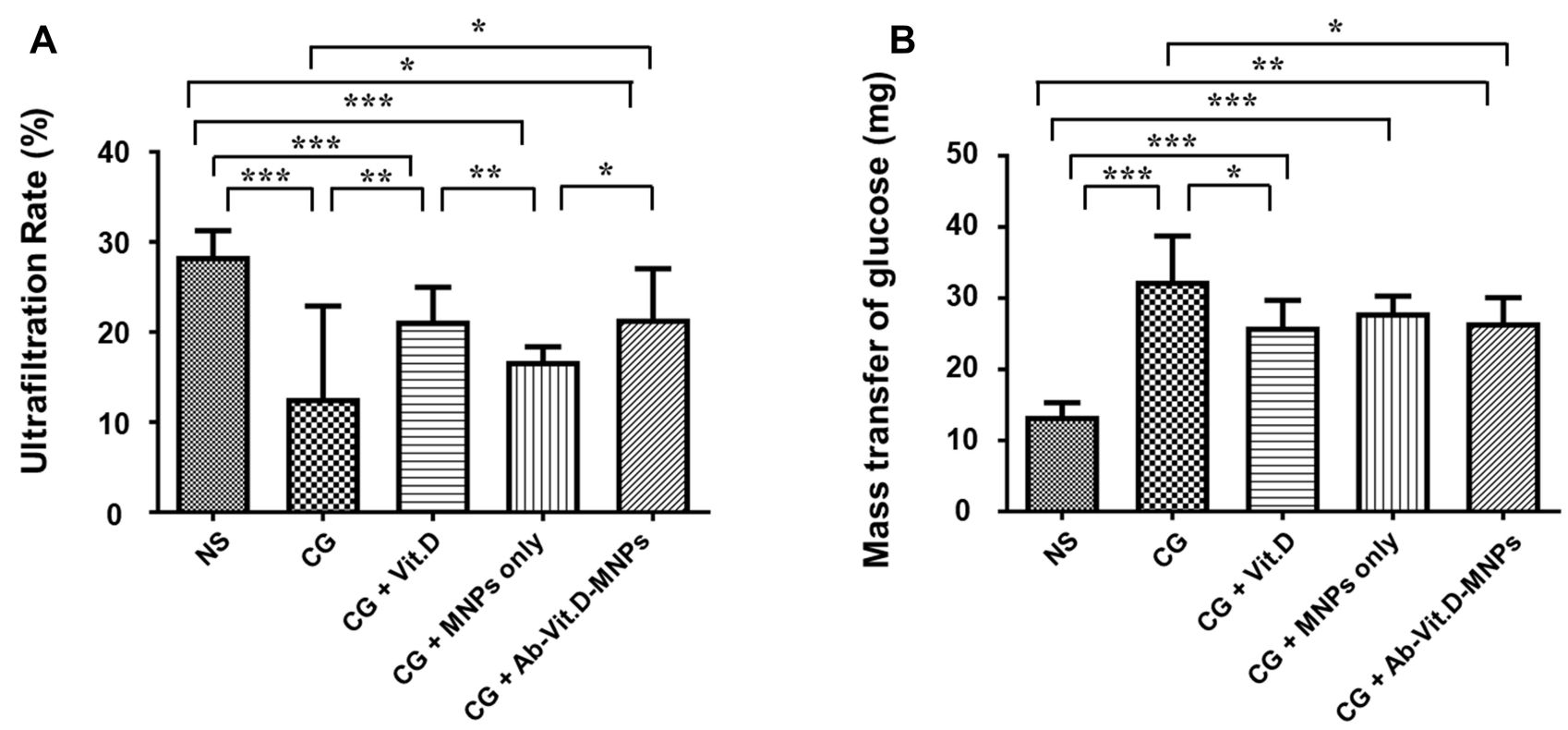

Figure 5 Ab-Vit.D-MNPs have the same therapeutic effect as vitamin D3 in ameliorating peritoneal functional deterioration in a peritoneal dialysis (PD) mouse model. (A) The ultrafiltration rate in the chlorhexidine gluconate (CG)-exposed group was significantly lower than that in the saline group; vitamin D3 corrected the CG-induced ultrafiltration decrease. The same therapeutic effect was observed in the Ab-Vit.D-MNP group. (B) The glucose mass transport also indicated that Ab-Vit.D-MNPs ameliorated the CG-induced increase in peritoneal permeability in the same way as vitamin $\mathrm{D}$. (Data are represented as the mean $\pm \mathrm{SD}, \mathrm{n} \geq 3 ; * \mathrm{P}<0.05$, **P $<0.0$ I, ***P $<$ $0.001)$.

\section{Ab-Vit.D-MNPs Have the Same Therapeutic Effect as Vitamin D3 in Ameliorating Peritoneal Fibrosis in a PD Animal Model}

Using the PD mouse model, we investigated the effect of Ab-Vit.D-MNPs on the structural deterioration of the peritoneal membrane (Figure 4A and B). Peritoneal fibrosis was significantly more severe in the CG-exposed group than in the saline group. Intraperitoneal administration of vitamin D3 significantly ameliorated the peritoneal fibrosis, as visualized by Masson's trichrome stain. The results showed that Ab-Vit.D-MNPs had the same therapeutic effect on peritoneal fibrosis as vitamin D.

\section{Ab-Vit.D-MNPs Have the Same Therapeutic Effect as Vitamin D3 in Ameliorating Peritoneal Functional Deterioration in a PD Animal Model}

To investigate the peritoneum function, a modified peritoneal equilibration test was performed. The ultrafiltration rate in the CG-exposed group was significantly lower than that in the saline group; vitamin D3 reduced the CGinduced ultrafiltration decrease. Ab-Vit.D-MNPs showed the same therapeutic effect (Figure 5A). The mass transport of glucose also indicated that Ab-Vit.D-MNPs could ameliorate the CG-induced increase in peritoneal permeability, similar to vitamin D3 (Figure 5B).

\section{Ab-Vit.D-MNPs Ameliorate the Side Effects of Vitamin D3 in a PD Mouse Model}

Vitamin D3 therapy is known to cause hypercalcemia and hyperphosphatemia, especially after overdose. We investigated the effect of Ab-Vit.D-MNPs on serum calcium and phosphate levels. The results showed that in the vitamin D3 group, serum calcium levels were significantly higher than those in the other groups; however, hypercalcemia was not detected in the Ab-Vit.D-MNP groups (Figure 6A). In the vitamin D3 group, serum phosphate levels were significantly lower than those in the other groups (Figure 6B). Further, we found poor food intake and significantly decreased $\mathrm{BW}$ in the vitamin $\mathrm{D} 3$ treatment group, but not in the Ab-Vit.D-MNP group (Figure 6C). A decrease in activity was also noted in the vitamin D3 treatment group but not in the Ab-Vit.D-MNP group.

\section{Discussion}

The therapeutic effects of vitamin D3 in PD-related peritoneal damage have been well established both in vitro and 

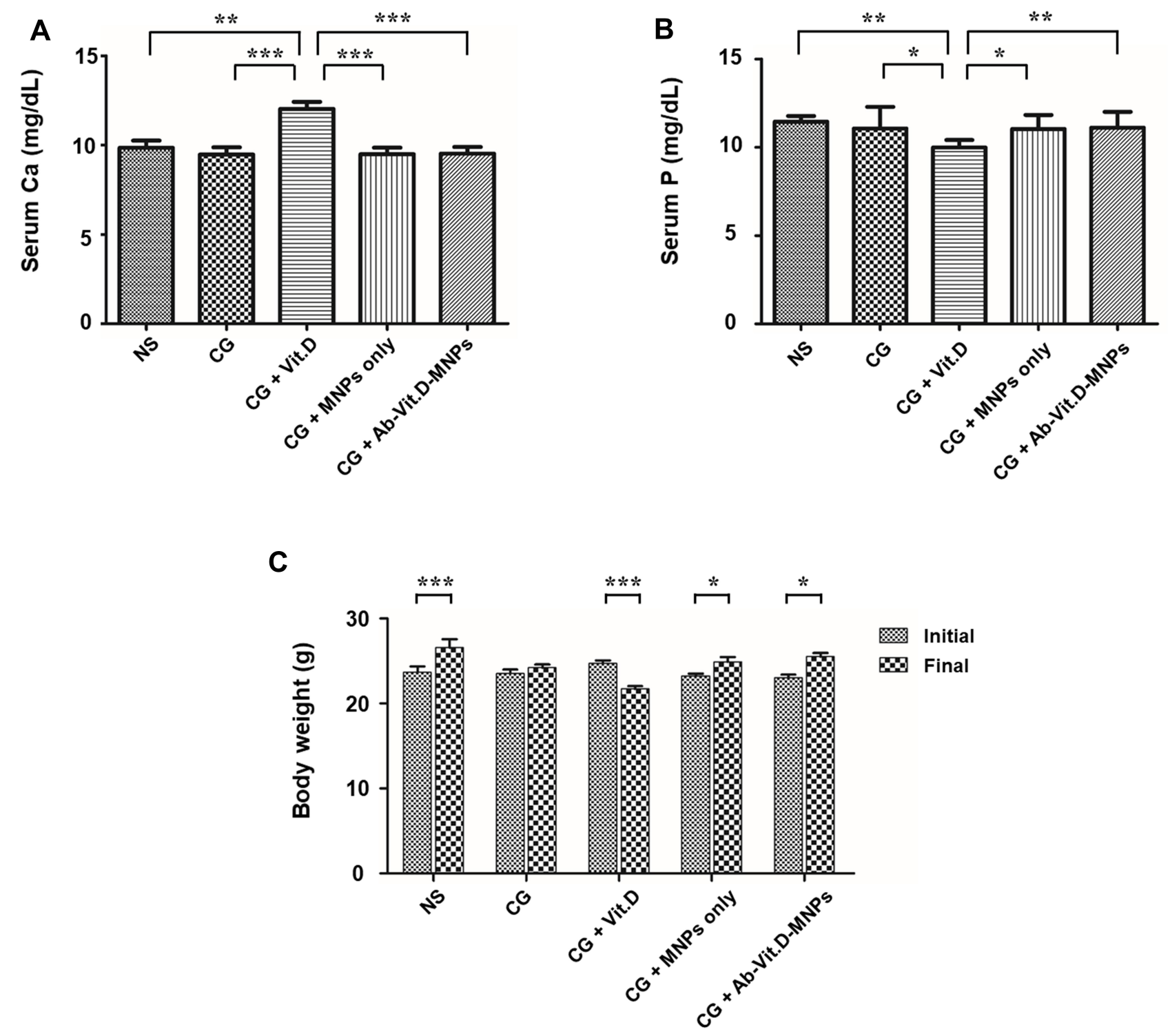

Figure 6 Ab-Vit.D-MNPs can ameliorate the side effects of vitamin D3 therapy in a peritoneal dialysis (PD) mouse model. (A) In the vitamin D3 group, serum calcium levels were significantly higher than those in the other groups; however, there was no hypercalcemia in the Ab-Vit.D-MNP group. (B) Serum phosphate levels were significantly lower in the vitamin D3 treatment group than in the Ab-Vit.D-MNP group. (C) Body weight (BW) was significantly decreased in the vitamin D3 treatment group but not in the Ab-Vit.D-MNP group. (Data are represented as the mean $\pm S D, n \geq 3 ; * P<0.05$, **P $<0.01$, *** $P<0.00 I$ ).

in vivo. ${ }^{3}$ However, using $1 \alpha, 25(\mathrm{OH})_{2} \mathrm{D}_{3}$ to treat peritoneal damage requires supra-physiological doses that induce side effects including hypercalcemia and hyperphosphatemia, ${ }^{3,19}$ limiting its clinical utility. In our previous study, we found that Ab-Vit.D-MNPs had the same therapeutic effect as vitamin D3 in vitro. ${ }^{13}$ Therefore, in this study, we investigated its therapeutic effect in vivo. The results showed that Vit.D-MNPs conjugated with an antibody against GPM6A, a peritoneal marker, enhanced peritoneum targeting. It showed the same therapeutic effect as vitamin D3 in ameliorating peritoneal fibrosis and peritoneal functional deterioration in our PD mouse model. Most importantly, it reduced the side effects of vitamin D3 overdosage, including hypercalcemia and BW loss.

In our study, we showed that these NPs are stable, biocompatible, and safe. One important reason for selecting alg-MNPs to deliver vitamin D3 is that alginate and $\mathrm{Fe}_{3} \mathrm{O}_{4}$ are FDA-approved biomaterials for human use. Our previous study and this study all showed that alg-MNPs are mainly excreted through the kidneys. ${ }^{20}$ This indicated that alg-MNPs did not accumulate in the organs causing 
super-oxidation and damage. This characteristic is very important when considering the possible clinical applications of our NPs. ${ }^{21}$

Another important consideration is the occurrence of BW loss after supra-physiological doses of vitamin D3, possibly due to hypercalcemia, which causes nausea, poor food intake, and weakness. ${ }^{22}$ We believe that the hypophosphatemia in the vitamin D3 treatment group was also due to nausea and poor food intake.

This study has some limitations. First, in our PD animal model, we used $\mathrm{CG}$ as a chemical irritant, not dialysate, to induce peritoneal fibrosis and functional deterioration. At the same time, our mice were not uremic and the effect of uremic toxins on the peritoneum could not be measured. The CG model of peritoneal damage is simple and easy to use; however, it does not fully represent PD fluid installation. ${ }^{23}$ It should be noted that results obtained in $\mathrm{CG}$ mice may not translate to those obtained in PD fluid-installation mice; in the latter, the peritoneum changes are more subtle and may follow different patterns. However, previous studies have suggested that it is a feasible model for studying peritoneal damage. ${ }^{24-26}$ Second, we only checked serum calcium and phosphate levels, but we did not generate aortic calcium and phosphate content data that would provide another evidence for vitamin D side effects.

\section{Conclusion}

Our study showed that Ab-Vit.D-MNPs delivered vitamin D3 specifically to the peritoneum and decreased the side effects of supra-physiological doses of vitamin D3. These results suggest that vitamin D3 nano-DDS may be an ideal therapeutic option to treat $\mathrm{PD}$-related peritoneal damage in future.

\section{Abbreviations}

Ab-R6G-MNPs, glycoprotein M6A antibody-rhodamine 6G magnetic nanoparticles; Ab-Vit.D-MNPs, glycoprotein M6A, and vitamin D3 magnetic nanoparticles; BD, bodyweight; DLS, dynamic light scattering; GPM6A, glycoprotein M6A; HD, hemodialysis; HPLC, highperformance liquid chromatography; IVIS, in vivo imaging system; MNPs, magnetic nanoparticles; Nano-DDS, nano-drug delivery system; NPs, nanoparticles; PD, peritoneal dialysis; R6G, rhodamine 6G; Vit.D-MNPs, vitamin D3 magnetic nanoparticles.

\section{Acknowledgments}

We are indebted to Shin-Han Tseng for the critical discussion and partial execution of the study. This study was supported by EDAHP-109003, NCKUEDA-10810, NCKUEDA-10811 and NCKUEDA-10907 from the Research Foundation of E-DA Hospital and National Cheng Kung University, Taiwan.

\section{Disclosure}

The authors declare that they have no conflicts of interest.

\section{References}

1. Han SH, Lee SC, Ahn SV, et al. Improving outcome of CAPD: twenty-five years' experience in a single Korean center. Perit Dial Int. 2007;27:432-440. doi:10.1177/089686080702700411

2. Kawaguchi Y, Hasegawa T, Nakayama M, Kubo H, Shigematu T. Issues affecting the longevity of the continuous peritoneal dialysis therapy. Kidney Int Suppl. 1997;62:S105-7.

3. Lee YC, Hung SY, Liou HH, et al. Vitamin D can ameliorate chlorhexidine gluconate-induced peritoneal fibrosis and functional deterioration through the inhibition of epithelial-to-mesenchymal transition of mesothelial cells. Biomed Res Int. 2015;2015:595030. doi:10.1155/2015/595030

4. Nakamoto H, Kawaguchi Y, Suzuki H. Is technique survival on peritoneal dialysis better in Japan? Perit Dial Int. 2006;26:136-143. doi: $10.1177 / 089686080602600203$

5. Schaefer F, Klaus G, Muller-Wiefel DE, Mehls O. Current practice of peritoneal dialysis in children: results of a longitudinal survey. Mid European Pediatric Peritoneal Dialysis Study Group (MEPPS). Perit Dial Int. 1999;19(Suppl 2):S445-9. doi:10.1177/089686089901902S73

6. Woodrow G, Turney JH, Brownjohn AM. Technique failure in peritoneal dialysis and its impact on patient survival. Perit Dial Int. 1997;17:360-364. doi:10.1177/089686089701700411

7. Andreoli SP, Langefeld CD, Stadler S, Smith P, Sears A, West K. Risks of peritoneal membrane failure in children undergoing long-term peritoneal dialysis. Pediatr Nephrol. 1993;7:543-547. doi:10.1007/BF00852541

8. Davies SJ, Phillips L, Griffiths AM, Russell LH, Naish PF, Russell GI. What really happens to people on long-term peritoneal dialysis? Kidney Int. 1998;54:2207-2217. doi:10.1046/j.15231755.1998.00180.x

9. Davies SJ, Phillips L, Naish PF, Russell GI. Peritoneal glucose exposure and changes in membrane solute transport with time on peritoneal dialysis. J Am Soc Nephrol. 2001;12:1046-1051.

10. Krediet RT. The peritoneal membrane in chronic peritoneal dialysis. Kidney Int. 1999;55:341-356.

11. Nevozhay D, Kanska U, Budzynska R, Boratynski J. [Current status of research on conjugates and related drug delivery systems in the treatment of cancer and other diseases]. Postepy Hig Med Dosw. 2007;61:350-360. [Article in Polish].

12. Lee CJ, Subeq YM, Lee RP, Liou HH, Hsu BG. Calcitriol decreases TGF-beta1 and angiotensin II production and protects against chlorhexide digluconate-induced liver peritoneal fibrosis in rats. Cytokine. 2014;65:105-118. doi:10.1016/j.cyto.2013.10.003

13. Lee YC, Huang CT, Cheng FY, et al. The clinical implication of Vitamin $\mathrm{D}$ nanomedicine for peritoneal dialysis-related peritoneal damage. Int J Nanomed. 2019;14:9665-9675. doi:10.2147/IJN.S215717

14. Su CH, Tsai CY, Tomanek B, Chen WY, Cheng FY. Evaluation of blood-brain barrier-stealth nanocomposites for in situ glioblastoma theranostics applications. Nanoscale. 2016;8:7866-7870. doi:10.1039/C6NR00280C 
15. Rongjie FU, JLa YW. Fat-soluble vitamins analysis on an agilent ZORBAX eclipse PAH polymeric C18 bonded column. Agilent Technologies; 2010:5990-5342EN.

16. Ishii Y, Sawada T, Shimizu A, et al. An experimental sclerosing encapsulating peritonitis model in mice. Nephrol Dial Transplant. 2001;16:1262-1266. doi:10.1093/ndt/16.6.1262

17. Zhu F, Li T, Qiu F, et al. Preventive effect of Notch signaling inhibition by a gamma-secretase inhibitor on peritoneal dialysis fluid-induced peritoneal fibrosis in rats. Am $J$ Pathol. 2010;176:650-659. doi:10.2353/ajpath.2010.090447

18. Patil S, Gawali S, Patil S, Basu S. Synthesis, characterization and in vitro evaluation of novel vitamin D3 nanoparticles as a versatile platform for drug delivery in cancer therapy. $J$ Mater Chem $B$. 2013;1:5742. doi:10.1039/c3tb21176b

19. Ozkan B, Hatun S, Bereket A. Vitamin D intoxication. Turk $J$ Pediatr. 2012;54:93-98.

20. Tsai YC, Teng IL, Jiang ST, Lee YC, Chiou YY, Cheng FY. Safe nanocomposite-mediated efficient delivery of MicroRNA plasmids for autosomal dominant polycystic kidney disease (ADPKD) therapy. Adv Healthc Mater. 2019;8:e1801358. doi:10.1002/ adhm. 201801358
21. Kamaly N, He JC, Ausiello DA, Farokhzad OC. Nanomedicines for renal disease: current status and future applications. Nat Rev Nephrol. 2016;12:738-753.

22. Gardner EC Jr, Hersh T. Primary hyperparathyroidism and the gastrointestinal tract. South Med J. 1981;74:197-199. doi:10.1097/ 00007611-198102000-00019

23. Vlijm A, Sampimon DE, de Graaff M, Struijk DG, Krediet RT. Experimental peritoneal sclerosis models should not be based on chlorhexidine gluconate anymore. Nephron Exp Nephrol. 2011;117: e1-8. doi:10.1159/000319651

24. Yoh K, Ojima M, Takahashi S. Th2-biased GATA-3 transgenic mice developed severe experimental peritoneal fibrosis compared with Th1-biased T-bet and Th17-biased RORgammat transgenic mice. Exp Anim. 2015;64:353-362. doi:10.1538/expanim.15-0019

25. Nishino T, Ashida R, Obata Y, et al. Involvement of lymphocyte infiltration in the progression of mouse peritoneal fibrosis model. Ren Fail. 2012;34:760-766. doi:10.3109/0886022X.2012.676527

26. Io K, Nishino T, Obata Y, Kitamura M, Koji T, Kohno S. SAHA suppresses peritoneal fibrosis in mice. Perit Dial Int. 2015;35:246-258. doi:10.3747/pdi.2013.00089
International Journal of Nanomedicine

\section{Publish your work in this journal}

The International Journal of Nanomedicine is an international, peerreviewed journal focusing on the application of nanotechnology in diagnostics, therapeutics, and drug delivery systems throughout the biomedical field. This journal is indexed on PubMed Central, MedLine, CAS, SciSearch ${ }^{\mathbb{R}}$, Current Contents ${ }^{\mathbb{R}} /$ Clinical Medicine, $^{2}$

\section{Dovepress}

Journal Citation Reports/Science Edition, EMBase, Scopus and the Elsevier Bibliographic databases. The manuscript management system is completely online and includes a very quick and fair peer-review system, which is all easy to use. Visit http://www.dovepress.com/ testimonials.php to read real quotes from published authors. 